\title{
Static pure Lovelock black hole solutions with horizon topology $S^{(n)} \times S^{(n)}$
}

\author{
Naresh Dadhich ${ }^{a, b}$ and Josep M. Pons ${ }^{c}$ \\ ${ }^{a}$ Centre for Theoretical Physics, Jamia Millia Islamia, \\ New Delhi 110025, India \\ ${ }^{b}$ Inter-University Centre for Astronomy and Astrophysics, \\ Post Bag 4, Pune $41100 \%$, India \\ ${ }^{c}$ Departament d'Estructura $i$ Constituents de la Matèria and \\ Institut de Ciències del Cosmos (ICCUB), Facultat de Física, \\ Universitat de Barcelona, Diagonal 647, E-08028 Barcelona, Catalonia, Spain \\ E-mail: nkd@iucaa.ernet.in, pons@ecm.ub.edu
}

ABSTRACT: It is well known that vacuum equation of arbitrary Lovelock order for static spacetime ultimately reduces to a single algebraic equation, we show that the same continues to hold true for pure Lovelock gravity of arbitrary order $N$ for topology $\mathbf{S}^{(\mathbf{n})} \times \mathbf{S}^{(\mathbf{n})}$. We thus obtain pure Lovelock static black hole solutions with two sphere topology for any order $N$, and in particular we study in full detail the third and fourth order Lovelock black holes. It is remarkable that thermodynamical stability of black hole discerns between odd and even $N$, and consequently between negative and positive $\Lambda$ and it favors the former while rejecting the latter.

Keywords: Classical Theories of Gravity, Black Holes

ArXiv EPrint: 1503.00974 


\section{Contents}

1 Introduction 1

2 Equation of motion 3

3 Particular cases $\quad 6$

3.1 Case $N=1$ : Einstein black hole 6

3.2 Case $N=2$ : Gauss-Bonnet black hole 6

3.3 Case $N=3$ : third order pure Lovelock black hole 6

$\begin{array}{lll}\text { 3.3.1 The noncritical case } d_{0}>3 & 7\end{array}$

3.4 Case $N=4$ : fourth order pure Lovelock black hole 8

$\begin{array}{ll}3.5 & \text { The general pattern }\end{array}$

4 Comparison with one sphere case $\quad 10$

5 Thermodynamics $\quad 11$

$\begin{array}{lll}5.1 & \text { Entropy } & 11\end{array}$

$\begin{array}{ll}5.2 \text { Temperature } & 12 \\ 5.3 & \text { Stability }\end{array}$

$\begin{array}{lll}5.3 & \text { Stability } & 12\end{array}$

$\begin{array}{llr}6 & \text { Discussion } & 14\end{array}$

$\begin{array}{ll}\text { A Proof that }\left[\mathcal{L}_{N}\right]_{0} \text { is a derivative } & 15\end{array}$

$\begin{array}{ll}\text { B Proof of (2.7) } & 16\end{array}$

\section{Introduction}

For higher dimensional considerations of gravity, Lovelock generalization is the most natural and appropriate as equation of motion continues to remain second order despite Lagrangian being homogeneous polynomial in Riemann. This property singles out Lovelock from all other generalizations and it includes Einstein gravity in linear order $N=1$ where $N$ is order of Lovelock polynomial action. It is well known that Einstein gravity is kinematic in $d=2 N+1=3$ dimension which means Ricci equal to zero implies Riemann zero. In other words, Riemann is entirely determined in terms of Ricci. Could this property be true for all $N$; i.e. does there exist a higher order generalization of Riemann such that in all odd $d=2 N+1$ dimensions it is entirely determined by its Ricci? The answer is yes [1] for pure Lovelock gravity for which the action contains only one $N$ th order term, besides of course the cosmological constant $\Lambda$, in the action (there is no sum over $N$ ). We define [2] $N$ th order analogue of Riemann (trace of its Bianchi derivative yields analogue 
of Einstein tensor and agrees with the one that is obtained by varying $N$ th order Lovelock action) which is a polynomial in Riemann and its contraction giving corresponding Ricci, and so we have Lovelock Riemann and Ricci tensors. Then it turns out that whenever Lovelock Ricci vanishes in odd $d=2 N+1$ dimensions, so does Lovelock Riemann. That is, pure Lovelock gravity which includes Einstein gravity for $N=1$, is kinematic in all odd $d=2 N+1$ dimensions. It is Lovelock kinematic because it is Lovelock Riemann that vanishes and not the usual Riemann. Kinematicity so defined is thus a universal gravitational property in higher dimensions, and if it is to be respected always, for a given $N$ there are only two pertinent dimensions $d=2 N+1,2 N+2$. For $d<2 N+1$, it is vacuous while for $d>2 N+2$, it is next order $(N+1)$ th that should be considered else kinematicity property will be violated. We shall therefore adhere to universality of this property and shall emphasize the pure Lovelock case for $d=2 N+1,2 N+2$.

Spherically symmetric static black holes in Gauss-Bonnet (GB) gravity have been studied $[3,4]$ and in fact for Lovelock gravity in general $[5,6]$. It turns out that ultimately one needs only to solve an algebraic polynomial of degree $N$. To manage this polynomial and for other physical considerations, it was assumed $[7,8]$ that all coupling constants are given in terms of the unique ground state, $\Lambda$. This made the equation degenerate and hence could be trivially solved. On the other hand, if we consider pure Lovelock equation which has only one $N$ th order term with $\Lambda$, then the equation is derivative degenerate and again could be solved trivially [9]. In all this, black hole horizon was a sphere of constant curvature. Some authors have considered more general cases with arbitrary couplings for each Lovelock term, see $[10,11]$, though an exact solution for polynomial equation cannot be obtained beyond $N=4$.

The next generalization came through consideration of horizon topology being a product of two spheres. Now horizon space is non-maximally symmetric Einstein space which has non-zero Weyl curvature. However both Weyl and Riemann have vanishing covariant derivative. Its first example was given by Nariai metric [12, 13]. An interesting GB black hole solution with two sphere topology was obtained by Dotti and Gleiser [14] and subsequently its various features like uniqueness and stability were studied by several authors [15-18]. It turns out that non-zero Weyl curvature makes a non-trivial contribution in solution in terms of a constant which is negative for GB case. This implies that $\Lambda$ should always be present and positive and there also occurs non central singularity. To avoid that, a range is prescribed for black hole mass in terms of $\Lambda$ [19].

In this paper our main aim is to extend this framework to pure Lovelock of arbitrary order. As a matter of fact, our study goes beyond pure Lovelock case $(d=2 N+2)$ with horizon topology $\mathbf{S}^{(\mathbf{N})} \times \mathbf{S}^{(\mathbf{N})}$ to include any $d=2 d_{0}+2, d_{0} \geq N$ with horizon topology $\mathbf{S}^{\left(\mathbf{d}_{0}\right)} \times \mathbf{S}^{\left(\mathbf{d}_{0}\right)}$. That is, we solve Lovelock $\Lambda$-vacuum equation of arbitrary order $N$ for a static black hole with two sphere topology in a spacetime with even dimension $d \geq 2 N+2$. It is remarkable that algebraic character of ultimate equation is carried through for this enlarged framework as well. The product of two spheres produces solid angle deficits which cancel out each-other's contribution in Ricci tensor but not in Riemann. For $N>1$, there is always Riemann present in the equation and to cancel out solid angle deficit contribution to it, a constant, say $p$, is required which is zero for Einstein $N=1$ case. Further it turns 
out that $\Lambda$ and $p$ always bear opposite sign, the former is negative and positive according to $N$ being even and odd, and vice-versa for $p$. Whenever $N$ is even, $p<0, \Lambda$ must be present and positive. To avoid non central singularity and for existence of horizon, black hole mass has to lie in a range prescribed in terms of $\Lambda$. On the other hand for odd $N$ when $p>0$, there is no such constraint. It is remarkable that the similar discerning feature also emerges from thermodynamical stability considerations. It favors odd $N$ and $\Lambda<0$ as against even $N$ and $\Lambda>0$. It is remarkable that two sphere topology distinguishes between odd and even $N$ and consequently negative and positive $\Lambda$ quite clearly. This is a new realization that has come up only when we have considered both odd and even $N>1$.

The paper is organized as follows: in next section we set up equation of motion for pure Lovelock $\Lambda$-vacuum of arbitrary order $N$ for a static metric with two sphere topology, and obtain general black hole solution. It is followed by consideration of particular cases for $N=1,2,3,4$. In section 4 we compare two sphere black holes with the corresponding one sphere ones while section 5 is devoted to thermodynamical considerations. We end up with discussion.

\section{Equation of motion}

Consider static metric configuration with product horizon space, $\mathbf{S}^{\left(\mathbf{d}_{\mathbf{0}}\right)} \times \mathbf{S}^{\left(\mathbf{d}_{\mathbf{0}}\right)}$ having symmetry group $\mathbf{S O}\left(\mathbf{d}_{\mathbf{0}}+\mathbf{1}\right) \times \mathbf{S O}\left(\mathbf{d}_{\mathbf{0}}+\mathbf{1}\right)$,

$$
d s^{2}=-A(r) d t^{2}+\frac{1}{A(r)} d r^{2}+r^{2}\left(d S_{\left(d_{0}\right)}^{2}+d S_{\left(d_{0}\right)}^{2}\right)
$$

describing an even-dimensional spacetime with dimension $d=2 d_{0}+2$, where $d S_{\left(d_{0}\right)}^{2}$ is metric of $d_{0}$ dimensional unit sphere. We compute Riemann tensor for the metric and its nonvanishing components are as follows:

$$
\begin{aligned}
& D(0,1)=R_{01}^{01}=-\frac{1}{2} A^{\prime \prime}(r) \\
& D(0, i)=R_{0 i}^{0 i}=-\frac{1}{2 r} A^{\prime}(r) \\
& D\left(0, i^{\prime}\right)=R_{0 i^{\prime}}{ }^{0} i^{\prime}=-\frac{1}{2 r} A^{\prime}(r) \\
& D(1, i)=R_{1}{ }^{1 i}=-\frac{1}{2 r} A^{\prime}(r) \\
& D\left(1, i^{\prime}\right)=R_{1 i^{\prime}}{ }^{i^{\prime}}=-\frac{1}{2 r} A^{\prime}(r) \\
& D(i, j)=R_{i j}{ }^{i j}=\frac{1}{r^{2}}(1-A(r)) \\
& D\left(i^{\prime}, j^{\prime}\right)=R_{i^{\prime} j^{\prime}} j^{\prime} j^{\prime}=\frac{1}{r^{2}}(1-A(r)) \\
& D\left(i, i^{\prime}\right)=R_{i i^{\prime}} i^{\prime}=-\frac{1}{r^{2}} A(r)
\end{aligned}
$$

where indices 0,1 refer to coordinates $t, r$, and indices $i, i^{\prime}$ to angle coordinates of two spheres. Notice that there is a crucial difference, in fact the only one, between the 
one-sphere case, $d S_{(d-2)}^{2}$, and our present two-spheres case, which is the last entry, $D\left(i, i^{\prime}\right)$, taking indices from two spheres. All the other components are the same for both configurations.

For this pure Lovelock Lagrangian of order $N$, we will show that the equation of motion (EOM) for the metric (2.1) is ultimately given by a single algebraic equation. Lovelock Lagrangian of arbitrary order $N$ is given by

$$
\mathcal{L}_{N}=\sqrt{-g} \delta_{\rho_{1} \ldots \rho_{N} \sigma_{1} \ldots \sigma_{N}}^{\mu_{1} \ldots \nu_{N} \ldots \nu_{N}} R_{\mu_{1} \nu_{1}}^{\rho_{1} \sigma_{1}} \ldots R_{\mu_{N} \nu_{N}}^{\rho_{N} \sigma_{N}}-\sqrt{-g} \tilde{\Lambda}
$$

which leads to EOM

$$
\left[\mathcal{L}_{N}\right]_{\rho}^{\mu}=\sqrt{-g} \delta_{\rho \rho_{1} \ldots \rho_{N} \sigma_{1} \ldots \sigma_{N}}^{\mu \mu_{1} \ldots \mu_{N} \nu_{1} \ldots \nu_{N}} R_{\mu_{1} \nu_{1}}^{\rho_{1} \sigma_{1}} \ldots R_{\mu_{N} \nu_{N}}^{\rho_{N} \sigma_{N}}-\sqrt{-g} \delta_{\rho}^{\mu} \tilde{\Lambda}=0,
$$

where $\tilde{\Lambda}$ is, up to some combinatorial factor, the cosmological constant. We keep the factor $\sqrt{-g}$ for reasons of later convenience.

Our aim is to implement eq. (2.2) into EOM (2.4) to get a differential equation for $A(r)$. It is obvious that for non-vacuous EOM, $\mu=\rho$, and we thus write $\left[\mathcal{L}_{N}\right]_{\mu}^{\mu}=:\left[\mathcal{L}_{N}\right]_{\mu}$. From eq. (2.2), it is clear that

$$
\left[\mathcal{L}_{N}\right]_{1}=\left[\mathcal{L}_{N}\right]_{0}
$$

whereas for angles $\mu=i, i^{\prime}$

$$
\left[\mathcal{L}_{N}\right]_{i}=\left[\mathcal{L}_{N}\right]_{i^{\prime}}=:\left[\mathcal{L}_{N}\right]_{i}, \forall i, i^{\prime}
$$

So we are left with two equations, $\left[\mathcal{L}_{N}\right]_{0}=0$ and $\left[\mathcal{L}_{N}\right]_{i}=0$. With some manipulations, which we leave for appendices $\mathrm{A}$ and $\mathrm{B}$, it can be shown that the former is in fact a total derivative (appendix A) while the latter takes the form (appendix B),

$$
\left[\mathcal{L}_{N}\right]_{i}=\frac{r}{2 d_{0}} \partial_{r}\left(\left[\mathcal{L}_{N}\right]_{0}\right)
$$

An equation of this kind is indeed expected on the basis of Noether identities for gauge theories. Note that here the gauge freedom is general covariance. In the case $N=1$ Noether identity eq. (2.7) is the contracted Bianchi identity for Einstein tensor. Thus we need to consider only the equation $\left[\mathcal{L}_{N}\right]_{0}=0$.

What is truly noteworthy is that $\left[\mathcal{L}_{N}\right]_{0}$ is a total derivative. It is well known that this is already the case with one sphere topology $[5,6]$. It is remarkable that this interesting feature carries forward also to the present case of two sphere topology (See appendix A). Now the $\Lambda$-free term in eq. (2.4) for $\left[\mathcal{L}_{N}\right]_{0}$ has the form $\frac{\partial}{\partial r}\left(r^{2 d_{0}-2 N+1} P_{N}(A(r))\right)$, where $P_{N}(A(r))$ is a polynomial of degree $N$. Here the $r^{2 d_{0}}$ factor has its origin in the density $\sqrt{-g}$. Then the equation, $\left[\mathcal{L}_{N}\right]_{0}=0$ from eq. (2.4) becomes

$$
\frac{\partial}{\partial r}\left(r^{2 d_{0}-2 N+1} P_{N}(A(r))\right)=r^{2 d_{0}} \tilde{\Lambda}
$$

which trivially integrates (with the appropriate redefinition $\Lambda=\frac{\tilde{\Lambda}}{2 d_{0}+1}$ ) to give

$$
P_{N}(A(r))=\Lambda r^{2 N}+\frac{M}{r^{2 d_{0}-2 N+1}},
$$


where $M$ is an integration constant. Note that in asymptotic expansion, the potential due to $M$ will go as $M / r^{2 d_{0}-1}=M / r^{d-3}$, which is the Schwarzschild potential in $d$ dimension. This formally indicates that $M$ is mass of the configuration. This is a general feature of pure Lovelock gravity that even though EOM is free of Einstein term yet the potential due to mass approaches asymptotically the Schwarzschild potential in the corresponding dimension [20].

As emphasized earlier, it is very remarkable that the general solution can now be obtained by simply solving the above algebraic equation of degree $N$.

The polynomial $P_{N}(A(r))$ takes the following form,

$$
P_{N}(A):=\sum_{l=0}^{N}\left(\frac{d_{0} !}{\left(d_{0}-l\right) !}\right)^{2} C\left[N-l, d_{0}-l\right](-A)^{l}(1-A)^{N-l} .
$$

with the combinatorial object

$$
C[m, s]=\frac{1}{2^{m}} \sum_{k=0}^{m}\left(\begin{array}{c}
m \\
k
\end{array}\right) \frac{s !}{(s-2 m+2 k) !} \frac{s !}{(s-2 k) !} .
$$

(An extension of the factorial to negative numbers is understood in this formula (2.11), using the gamma function. In particular notice that $C[N, N]$ vanishes for odd $N$ )

This polynomial can also be expressed alternatively as

$$
\begin{aligned}
P_{N}(A)=\sum_{l=0}^{N} & \sum_{k=0}^{N-l} \frac{1}{2^{N-l}} \frac{N !}{k ! l !(-k-l+N) !} \\
& \frac{d_{0} !}{\left(d_{0}-2 k-l\right) !} \frac{d_{0} !}{\left(d_{0}+2 k-2(N-l)-l\right) !}(-A(r))^{l}(1-A(r))^{N-l} .
\end{aligned}
$$

Let us write

$$
A(r)=\frac{d_{0}-1}{2 d_{0}-1}(1-\Psi(r))
$$

and define $Q_{N}(\Psi)=P_{N}(A)$. With this substitution the algebraic equation (2.9) becomes

$$
Q_{N}(\Psi):=\Lambda r^{2 N}+\frac{M}{r^{2 d_{0}-2 N+1}},
$$

The substitution (2.13) has the specific property that the polynomial $Q_{N}(\Psi)$ is now free of $\Psi^{N-1}$ term. This means that sum of its $N$ roots $\Psi_{l}, l=1, \cdots N$ is zero.

In terms of $\Psi$, the metric is written as

$$
d s^{2}=-(1-\Psi(r)) d t^{2}+\frac{1}{1-\Psi(r)} d r^{2}+\frac{d_{0}-1}{2 d_{0}-1} r^{2}\left(d S_{\left(d_{0}\right)}^{2}+d S_{\left(d_{0}\right)}^{2}\right) .
$$

Eq. (2.14) is the general algebraic equation for pure Lovelock black hole of arbitrary order $N$. Next we consider specific cases for $N=1,2,3,4$ to show that the known Einstein and Gauss-Bonnet black holes [3, 4] are all included in this equation. 


\section{Particular cases}

\subsection{Case $N=1$ : Einstein black hole}

In this case, eq. (2.8) takes the form

$$
r^{2 d_{0}-1} \Psi(r)=\Lambda r^{2 d_{0}+1}+M
$$

giving

$$
\Psi(r)=\Lambda r^{2}+\frac{M}{r^{2 d_{0}-1}}
$$

for the metric (2.15). This is the usual Schwarzschild-dS/AdS solution except for constant factor $\frac{d_{0}-1}{2 d_{0}-1}$ before two spheres metric. Note that this factor causes solid angle deficit for each sphere but the two together conspire to cancel out each-other in Ricci giving rise to $\Lambda$-vacuum. This feature was already noticed by [21]. However this cancellation cannot carry forward to higher order Lovelock simply because analogue of Ricci or Einstein tensor for higher order Lovelock would also involve Riemann. Their contributions to Riemann do not however cancel out each-other. For that an additional constant would be required for $N>1$ solutions as as we would see in the cases that follow.

\subsection{Case $N=2$ : Gauss-Bonnet black hole}

In Gauss-Bonnet case, the substitution (2.13) turns out to be very efficient, making the polynomial (2.10) proportional to $\Psi^{2}(r)+\frac{d_{0}}{\left(d_{0}-1\right)^{2}\left(2 d_{0}-3\right)}$. Then the solution for eq. (2.8) is of the form

$$
\Psi(r)= \pm \sqrt{p+\Lambda r^{4}+\frac{M}{r^{2 d_{0}-3}}} .
$$

where

$$
p=-\frac{d_{0}}{\left(d_{0}-1\right)^{2}\left(2 d_{0}-3\right)}
$$

We choose the upper positive sign in solution (3.2) for existence of black hole horizon as well as for gravity being attractive. Note here the constant $p$ under the radical is negative and it is the one that compensates the term coming from square of Riemann in GB Ricci analogue which is quadratic in Ricci and Riemann. As argued earlier for Einstein case that solid angle deficits of the two spheres cancel out each other to leave $\Lambda$-vacuum intact while in the case of GB, there is also a quadratic term in Riemann, it is that which requires this additional constant. For pure GB black hole, we have $d=2 N+2=6, d_{0}=2$ and $p=-2$. This case has been comprehensively discussed in [19]. That is $\Lambda$ and $p$ would always bear opposite sign and they are respectively positive and negative for even and odd $N$ and vice-versa. We shall verify this feature in the following cases for $N=3,4$.

\subsection{Case $N=3$ : third order pure Lovelock black hole}

With the appropriate redefinitions, always preserving the signs of $\Lambda$ and $M$, eq. (2.9) is now,

$$
a \Psi(r)^{3}+b \Psi(r)-p=\Lambda r^{6}+\frac{M}{r^{2 d_{0}-5}}
$$


with

$$
\begin{aligned}
a & =\left(d_{0}-1\right)^{3}\left(2 d_{0}-5\right)\left(2 d_{0}-3\right)>0 \\
b & =3\left(d_{0}-1\right) d_{0}\left(2 d_{0}-5\right)>0 \\
p & =2 d_{0}\left(2 d_{0}+1\right)>0
\end{aligned}
$$

The critical dimension (pure Lovelock) for $N=3$ is $d=2 N+2=8, d_{0}=3$, and so we write (after redefining $\Lambda$ and $M$ to absorb a factor of 6 )

$$
4 \Psi(r)^{3}+3 \Psi(r)=7+\Lambda r^{6}+\frac{M}{r} .
$$

For existence of horizon, $\Psi\left(r_{h}\right)=1$, we need $\Lambda r_{h}^{6}+\frac{M}{r_{h}}=0$ which gives $M=-\Lambda r_{h}^{7}$. The equation then becomes

$$
4 \Psi(r)^{3}+3 \Psi(r)=7+\frac{\Lambda}{r}\left(r^{7}-r_{h}^{7}\right) .
$$

It is clear that $4 \Psi^{3}+3 \Psi$ is monotonically increasing. To keep $\Psi<1$ (i.e, $A(r)>0$ ), which in eq. (3.6) means r.h.s. $<7$, we need $\frac{\Lambda}{r}\left(r^{7}-r_{h}^{7}\right)<0$. For $r>r_{h}$, this means that $\Lambda$ must be negative $\Lambda<0$ which also ensures $M>0$ in $r_{h}=\left(\frac{M}{-\Lambda}\right)^{\frac{1}{7}}$. The solution $\Psi(r)$ takes the form

$$
\Psi(r)=\frac{\left(\sqrt{S^{2}+1}+S\right)^{2 / 3}-1}{2\left(\sqrt{S^{2}+1}+S\right)^{1 / 3}},
$$

with $S=7-\frac{|\Lambda|}{r}\left(r^{7}-r_{h}^{7}\right)$. Note that $r>r_{h} \Rightarrow S<7 \Rightarrow \Psi(r)<1$. This describes the region outside black hole horizon. Thus there is no constraint on horizon radius and mass is given by $M=|\Lambda| r_{h}^{7}$.

The limit $r_{h} \rightarrow 0(\Rightarrow M \rightarrow 0)$ is regular for $\Psi(r)$ but then the background develops a naked singularity at $r=0$. In fact the Kretschmann scalar, $K=R_{\mu \nu \rho \sigma} R^{\mu \nu \rho \sigma}$, behaves for $r \rightarrow 0$ as

$$
K \sim \frac{24}{r^{4}}
$$

Note also that in the $r \rightarrow \infty$ limit, $\Psi(r) \rightarrow-\frac{|\Lambda|^{1 / 3}}{2^{2 / 3}} r^{2}$. As mentioned earlier, $p=7>0$ and $\Lambda$ is required to be negative. On the other hand for $N=2 \mathrm{~GB}$ case, it was the reverse, $p=-2<0$ and $\Lambda>0$. That is, $\Lambda$ has always to be non-zero, positive for even $N$ and negative for odd $N$ while for $N=1$ it could be both positive and negative. Pure Lovelock critical dimension for $N=1$ is $d=4$ for which $d_{0}=1$ that makes $S^{(1)} \times S^{(1)}$ space flat. Therefore dimension has always to be $>4$ and hence Einstein case in this setting is not a proper pure Lovelock critical dimension case. We will also see in the next case of $N=4$ that $\Lambda>0$ and $p<0$.

\subsubsection{The noncritical case $d_{0}>3$}

The $N=3$ case, for $d_{0}>3$ (non pure Lovelock) allows for a window of positive $\Lambda$. Although it is not the case that really interests us (which is $d_{0}=N=3$ ), we will discuss it here for the shake of completeness. 


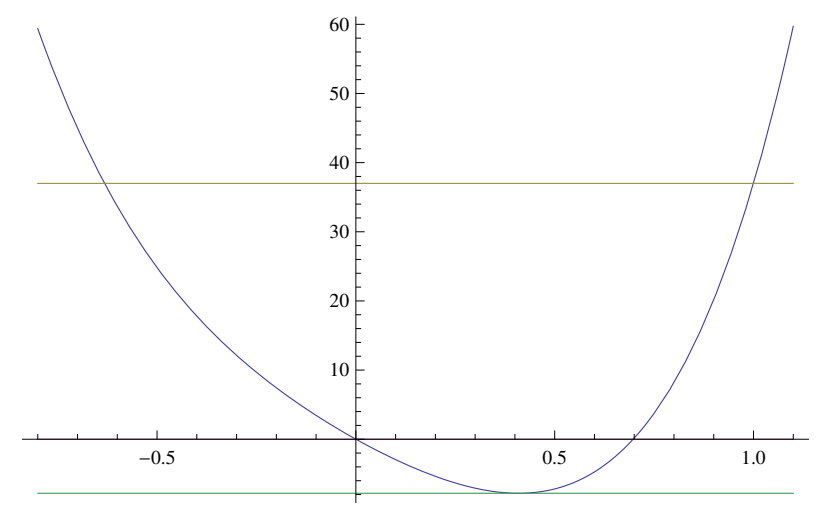

Figure 1. Plot of $F(\Psi)$ (eq. (3.11)). Horizontal lines are $y=F(1)=37$ and $y=F\left(\Psi_{0}\right) \simeq-7.81$ at the minimum $\Psi_{0} \simeq 0.41$.

Let us assume $d_{0}=4$, which is representative of the general $d_{0}>3$ case. Equation (3.4) is (an irrelevant positive factor has been eliminated from the definition (2.14))

$$
Q_{3}(\Psi(r))=\Lambda r^{6}+\frac{M}{r^{3}}
$$

What happened in the critical, pure Lovelock case was that at the horizon $\left(\Psi\left(r_{h}\right)=1\right)$ the polynomial $Q_{3}$ vanished (the reason is that $P_{N}(0)=0$ for odd $\left.d_{0}=N\right)$. Now instead we have $Q_{3}(1)>0$. and the polynomial is monotonically increasing, $Q_{3}^{\prime}(\Psi)>0$.

At the horizon we have $Q_{3}(1)\left(=P_{3}(0)\right)=\Lambda r_{h}^{6}+\frac{M}{r_{h}^{3}}$. Thus we can get $M=P_{3}(0) r_{h}^{3}-$ $\Lambda r_{h}^{6}$ and so we write (3.8)) as

$$
Q_{3}(\Psi(r))=\Lambda\left(r^{6}-\frac{r_{h}^{9}}{r^{3}}\right)+P_{3}(0) \frac{r_{h}^{3}}{r^{3}} .
$$

Since $\Psi\left(r_{h}\right)=1$, for $r>r_{h}$ but close enough to the horizon, we need $\Psi(r)<1$, otherwise $A(r)$ turns negative. Using that the polynomial $Q_{3}(\Psi)$ is monotonically increasing, this means that we need, at the horizon, $\frac{d}{d r} Q_{3}(\Psi(r))_{\left.\right|_{r_{h}}}<0$ which, in terms of the r.h.s. of (3.9), yields the condition

$$
\Lambda<\frac{P_{3}(0)}{3 r_{h}^{6}} .
$$

Thus there is a window for positive $\Lambda$. Of course any negative value for $\Lambda$ is admissible and in such case the analysis continues along the same path as the critical $d_{0}=N=3$ case. The case with positive $\Lambda$ can be parametrized by taking $\Lambda=\frac{P_{3}(0)}{(3+a) r_{h}^{6}}$ with $a>0$. We then find that there is a minimum $r_{0}$ for $\Psi(r)\left(\Psi^{\prime}\left(r_{0}\right)=0\right)$ at $r_{0}=\left(1+\frac{a}{2}\right)^{\frac{1}{9}} r_{h}>r_{h}$. Then, for $r>r_{0} \Psi^{\prime}(r)$ turns positive and we obtain a second, cosmological deSitter-like horizon at $r_{c}$ when $\Psi\left(r_{c}\right)=1$.

\subsection{Case $N=4$ : fourth order pure Lovelock black hole}

Again with the appropriate redefinitions of $\Lambda$ and $M$, eq. (2.9) takes the form.

$$
a \Psi(r)^{4}+b \Psi(r)^{2}+c \Psi(r)-p=\Lambda r^{8}+\frac{M}{r^{2 d_{0}-7}}
$$


with

$$
\begin{aligned}
a & =\left(d_{0}-1\right)^{4}\left(2 d_{0}-7\right)\left(2 d_{0}-5\right)\left(2 d_{0}-3\right)>0, \\
b & =6\left(d_{0}-1\right)^{2} d_{0}\left(2 d_{0}-7\right)\left(2 d_{0}-5\right)>0, \\
c & =-8\left(d_{0}-1\right) d_{0}\left(2 d_{0}-7\right)\left(2 d_{0}+1\right)<0, \\
p & =-3 d_{0}\left(d_{0}\left(10 d_{0}+3\right)+2\right)<0 .
\end{aligned}
$$

All cases for $d_{0} \geq 4$ are similar and we focus on the pure Lovelock $N=4, d_{0}=4$ case. Redefining again for convenience $\Lambda$ and $M$, the equation for $\Psi(r)$ becomes

$$
F(\Psi(r)) \equiv 45 \Psi(r)^{4}+24 \Psi(r)^{2}-32 \Psi(r)=-\frac{232}{3}+\frac{\Lambda}{8} r^{8}+\frac{M}{r} .
$$

The plot of $F(\Psi)$ against $\Psi$ (see figure 1) has a parabola like shape with a minimum at $\Psi_{0} \simeq 0.41$, where $F\left(\Psi_{0}\right)$ takes the approximate value $F\left(\Psi_{0}\right) \simeq-7.81$. We need to find a region for $r$ for which $\Psi(r)<1$. Note that $F(1)=37$. Thus we need values of $r$ such that, approximately

$$
-7.81<-\frac{232}{3}+\frac{\Lambda}{8} r^{8}+\frac{M}{r}<37,
$$

or

$$
69.2<\frac{\Lambda}{8} r^{8}+\frac{M}{r}<114.3 .
$$

Clearly this inequality requires $\Lambda>0$ while $M$ is always to be positive. Otherwise one of the bounds on $r$ will be an unwanted naked singularity, $\Psi(r)$ turning complex. In fact the minimum for $r$ should respect the range given in (3.12). The minimum is at $r_{0}=\left(\frac{M}{\Lambda}\right)^{\frac{1}{9}}$, and requiring that it satisfies the bounds (3.12), we end up with following range for $\Lambda$ and $M$,

$$
61.8<M^{\frac{8}{9}} \Lambda^{\frac{1}{9}}<101,6 .
$$

For $\Lambda$ and $M$ satisfying this bound, we can find a region $r_{h}<r<r_{c}$ such that $\Psi\left(r_{h}\right)=$ $\Psi\left(r_{c}\right)=1$ where $r_{h}$ is black hole and $r_{c}$ cosmological horizon (similar to deSitter), and $r_{0}$ also satisfies $r_{h}<r_{0}<r_{c}$. Note that here $p=-232 / 3$ and $\Lambda>0$ bearing out the general pattern as envisaged earlier.

\subsection{The general pattern}

As argued in Introduction, the distinguishing property of pure Lovelock gravity is its kinematicity in odd $d+2 N+1$ dimension. For this property to be universal; i.e. true for all odd $d=2 N+1$ dimensions, the equation should be valid only in the two critical odd and even dimensions, $d=2 N+1,2 N+2$. We shall therefore stick to the critical, pure Lovelock case, with $d_{0}=N$. Based on the cases $N=2,3,4$ and more (we have explored up to $N=12$ but nothing prevents from going to any higher $N$ ), there emerges the following general pattern that $\Lambda$ and $p$ (note that up to a positive factor which will be absorbed in $\Lambda$ and $M$, $p$ is defined as $\left.p=-Q_{N}(0)\right)$ always bear opposite sign, the former is respectively positive and negative for even and odd values of $N$ while the opposite is true for the latter. For $N$ even the shape of $Q_{N}(\Psi)$ is parabola with a single minimum $\Psi_{m}$ satisfying $0<\Psi_{m}<1$, 
similar to the case $N=4$ as shown in in figure 1. For $N$ odd $Q_{N}(\Psi)$ is a monotonically increasing function.

It may be noted that the parameter $p$ arises from countering the deficit angle contribution in the Riemann tensor and hence its sign and value is determined by this geometric feature. On the other hand for even $N, \Lambda>0$ is required for the polynomial (2.14) to have a minimum while for odd $N$, for some $r, Q_{N}(\Psi)<C[N, N]=0$ in the critical $d_{0}=N$ dimension, and for which $\Lambda$ has to be negative.

As expected whenever $\Lambda>0$ ( $N$ even), there exist both black hole and cosmological deSitter-like horizons, and a range of values for black hole mass in terms of $\Lambda$. When $\Lambda<0$ ( $N$ odd), there is only black hole horizon without any constraint on mass (See section 5 for non pure Lovelock cases). As a matter of fact, since $C[N, N]=0$ for $N$ odd, we will have $Q_{N}(1)=0$ for odd $d_{0}=N$ because $Q_{N}(1)=P_{N}(0)=C\left[N, d_{0}\right]$. Thus it is both $\mathrm{BH}$ and cosmological horizons in $d=6,10,14, \ldots$ and only one $\mathrm{BH}$ horizon in $d=8,12,16, \ldots$ dimensions.

\section{Comparison with one sphere case}

It is well known that static spherically symmetric vacuum equation for the general Lovelock Lagrangian ultimately reduces to an algebraic equation $[5,6]$. The difference between one and two spheres configurations is the absence of the terms with $i^{\prime}$ indices for the former in (2.2). This fact greatly simplifies the analysis and it follows on the same lines as in our case. The analogue of of the polynomial (2.10) becomes just a monomial in $(1-A)$, namely $P_{N}(A)^{(1)}:=\frac{(d-2) !}{(d-2 N-2) !}(1-A)^{N}$. The combinatorial factor is customarily absorbed in the general Lovelock Lagrangian $\mathcal{L}_{\text {Lov. }}=\sum_{N} c_{N} \mathcal{L}_{N}$ with a redefinition of coefficients $c_{N}$. Writing $A(r)=1-r^{2} B(r)$, we have the analogue of EOM (2.8) as

$$
\frac{\partial}{\partial r}\left(r^{2 d_{0}+1} B(r)^{N}\right)=r^{2 d_{0}} \Lambda
$$

If the Lagrangian is a sum of several Lovelock terms $\sum_{N=1}^{N_{0}} c_{N} \mathcal{L}_{N}$, then $B(r)^{N}$ in (4.1) is substituted by $\sum c_{N} B(r)^{N}$ and we have, on integrating (4.1),

$$
\sum_{N=0}^{N_{0}} c_{N} B(r)^{N}=\frac{M}{r^{2 d_{0}+1}}
$$

where the coefficient $c_{0}$ is proportional to $-\Lambda$ and the integration constant $M$ is proportional to black hole mass.

This nice simplification in eq. (4.2), due to the fact that the power of the variable $r$ in the bracket in the l.h.s. of (4.1) does not depend on $N$, is no longer available in the twospheres case because of the different structure of the polynomial. The crucial difference in the two cases is the presence of terms of the type $D\left(i, i^{\prime}\right)$ in eq. (2.2) which, unlike the terms $D(i, i)$ and $D\left(i^{\prime}, i^{\prime}\right)$, are not proportional to $1-A(r)$, but to $A(r)$. 


\section{Thermodynamics}

Even without knowing solution to eq. (2.9) explicitly, it is possible nevertheless to perform thermodynamical analysis for its black hole $(\mathrm{BH})$ solutions by just using EOM relying on the fact that these equations are of polynomial form. Therefore the method described below can be applied whenever EOM are of this type. Here we assume that solution $A(r)$ exist with a horizon $A\left(r_{h}\right)=0$, for some range of parameters $\Lambda$ and $M$. As a matter of fact we already know that such BHs exist for $N=1,2$ and we have shown in section 3 that this is indeed the case for arbitrary $N$.

Let us consider eq. (2.9) for a putative solution $A(r, M)$ (since $\Lambda$ plays no special role here we do not need to explicitate the dependence of $A$ on $\Lambda$ ). Thus we have the identity

$$
P_{N}(A(r, M))=\Lambda r^{2 N}+\frac{M}{r^{2 d_{0}-2 N+1}},
$$

and next we differentiate this identity w.r.t. $M$ and write We get

$$
\left.P_{N}^{\prime}(0) \frac{\partial A}{\partial M}\right|_{A=0}=\frac{1}{r^{2 d_{0}-2 N+1}} .
$$

We also note that $P_{N}^{\prime}(0)<0$ because the definition (2.10) gives

$$
P_{N}^{\prime}(0)=-N\left(d_{0}^{2} C\left[N-1, d_{0}-1\right]+C\left[N, d_{0}\right]\right)<0 .
$$

Thus

$$
\left.\frac{\partial A}{\partial M}\right|_{A=0}=-\frac{1}{\left|P_{N}^{\prime}(0)\right| r_{h}^{2 d_{0}-2 N+1}} .
$$

On the other hand, we can trade $r_{h}$ and $M$ for each other, which defines the function $M\left(r_{h}\right)$ so that $A(r, M(r))=0$ while varying $r$. Thus we can write the identity

$$
A(r, M(r))=0
$$

as implicitly defining the function $M(r)$. Its $r$-derivative gives the identity

$$
A^{\prime}(r, M(r))+\left.\frac{\partial A}{\partial M}\right|_{A=0} M^{\prime}(r)=0,
$$

where $A^{\prime}(r, M(r))$ is the derivative with respect to the first argument $r$. In fact the Euclidean method identifies $A^{\prime}(r, M(r))$ as the Hawking temperature $A^{\prime}(r, M(r))=4 \pi T(r)$. Thus we have

$$
\frac{M^{\prime}(r)}{T(r)}=-4 \pi \frac{1}{\left.\frac{\partial A}{\partial M}\right|_{A=0}}=4 \pi\left|P_{N}^{\prime}(0)\right| r^{2 d_{0}-2 N+1} .
$$

\subsection{Entropy}

The entropy is then obtained by integration of the First Law

$$
S=\int \frac{d M}{T}=\int_{0}^{r_{h}} \frac{M^{\prime}(r)}{T(r)} d r=\frac{4 \pi\left|P_{N}^{\prime}(0)\right|}{2 d_{0}-2 N+2} r_{h}^{2 d_{0}-2 N+2},
$$

where we have assumed the vanishing of the entropy when the horizon shrinks to zero. 
Since we only know that $M$ is proportional to the mass of the $\mathrm{BH}$, there is an undetermined numerical factor not included in (5.4). The relevant finding nonetheless is that $S$ is proportional to $r_{h}^{2 d_{0}-2 N+2}$. Note that in the critical Lovelock dimension, $d=2 N+2$, the entropy becomes proportional to $r^{2}$ or, since the area $\mathbf{A}$ of the horizon is proportional to $r_{h}^{2 d_{0}}=r_{h}^{2 N}$, it turns out that

$$
S_{\left(d_{0}=N\right)} \simeq \mathbf{A}^{\frac{1}{N}}
$$

which confirms that we are in the same universality class as in the spherical pure Lovelock $\mathrm{BH}$, as discussed in [9].

\subsection{Temperature}

The temperature con be obtained using the identity (5.1), in which $M$ is a fixed parameter. The $r$-derivative of (5.1), written at the horizon $r_{h}$ gives

$$
P_{N}^{\prime}(0) A^{\prime}\left(r_{h}, M\right)=2 N \Lambda r_{h}^{2 N-1}-\left(2 d_{0}-2 N+1\right) \frac{M}{r_{h}^{2 d_{0}-2 N+2}},
$$

which means that, since $P_{N}^{\prime}(0)<0$ and $A^{\prime}(r, M(r))=4 \pi T(r)$,

$$
T=\frac{1}{4 \pi\left|P_{N}^{\prime}(0)\right|}\left(\left(2 d_{0}-2 N+1\right) \frac{M}{r_{h}^{2 d_{0}-2 N+2}}-2 N \Lambda r_{h}^{2 N-1}\right)
$$

We can eliminate $\Lambda$ from this expression by taking into account that (5.1) implies, at the horizon,

$$
P_{N}(0)=\Lambda r_{h}^{2 N}+\frac{M}{r_{h}^{2 d_{0}-2 N+1}},
$$

(Note incidentally that $M\left(r_{h}\right)$ is trivially obtained from (5.5). Note also that the value $P_{N}(0)=C\left[N, d_{0}\right]$ is obtained from (2.10).)

Using (5.5) we obtain

$$
T=\frac{1}{4 \pi\left|P_{N}^{\prime}(0)\right|}\left(\left(2 d_{0}+1\right) \frac{M}{r_{h}^{2 d_{0}-2 N+2}}-\frac{2 N}{r_{h}} P_{N}(0)\right),
$$

which in the critical Lovelock dimension, $d=2 N+2$, gives

$$
T_{\left(d_{0}=N\right)}=\frac{1}{4 \pi\left|P_{N}^{\prime}(0)\right|}\left((2 N+1) \frac{M}{r_{h}^{2}}-\frac{2 N}{r_{h}} P_{N}(0)\right),
$$

which agrees, up to numerical factors, with the results obtained in [9] for the spherical pure Lovelock BH.

\subsection{Stability}

Here we follow the approach of refs. $[11,18]$. Local thermodynamical stability will correspond to positive specific heat, $C_{e}=\frac{d M}{d T}$. It is easy to compute it as

$$
C_{e}=\frac{M^{\prime}\left(r_{h}\right)}{T^{\prime}\left(r_{h}\right)} \text {. }
$$


Using eq. (5.5) to express $M$ in terms of $r_{h}$, we have

$$
M\left(r_{h}\right)=\left(P_{N}(0)-\Lambda r_{h}^{2 N}\right) r_{h}^{2 d_{0}-2 N+1} .
$$

Then temperature in eq. (5.6) is given by

$$
T=\frac{1}{4 \pi\left|P_{N}^{\prime}(0)\right|}\left(\left(2 d_{0}-2 N+1\right) \frac{P_{N}(0)}{r_{h}}-\left(2 d_{0}+1\right) \Lambda r_{h}^{2 N-1}\right) .
$$

To keep $M>0$, as required from the analysis in section 3 ,

$$
P_{N}(0)>\Lambda r_{h}^{2 N}
$$

Note that $P_{N}(0) \geq 0$ always. It only vanishes at the critical $d_{0}=N$ dimension for odd $N$. In all other cases $P_{N}(0)>0$. To keep $T>0$, we must have

$$
P_{N}(0)>\frac{\left(2 d_{0}+1\right)}{\left(2 d_{0}-2 N+1\right)} \Lambda r_{h}^{2 N} \text {. }
$$

Thus for $\Lambda<0$, the above constraints (5.9) and (5.10) impose no restrictions whereas for $\Lambda>0$ the constraint (5.10), which anyway implies (5.9), must be satisfied.

From eq. (5.3) it is clear that $T>0 \Leftrightarrow M^{\prime}>0$. To have local thermodynamical stability we therefore need $T^{\prime}\left(r_{h}\right)>0$. In fact, since

$$
T^{\prime}\left(r_{h}\right)=\frac{1}{4 \pi\left|P_{N}^{\prime}(0)\right|}\left(-\left(2 d_{0}-2 N+1\right) \frac{P_{N}(0)}{r_{h}^{2}}-\left(2 d_{0}+1\right)(2 N-1) \Lambda r_{h}^{2 N-2}\right),
$$

the condition $T^{\prime}\left(r_{h}\right)>0$ is equivalent to

$$
P_{N}(0)<-\frac{\left(2 d_{0}+1\right)(2 N-1)}{2 d_{0}-2 N+1} \Lambda r_{h}^{2 N}
$$

Local thermodynamical stability is thus guaranteed if the bounds (5.10) and (5.11) are satisfied. Since $P_{N}(0) \geq 0$, eq. (5.11) is inconsistent for positive $\Lambda \geq 0$, and hence it is ruled out. Thus we need $\Lambda<0$; i.e. $N$ odd. If in addition $d_{0}=N, P_{N}(0)=0$ and therefore no restrictions of any kind. If $P_{N}(0)>0$, there is a lower bound (eq. (5.11)) on BH size,

$$
r_{h}^{2 N}>\frac{2 d_{0}-2 N+1}{\left(2 d_{0}+1\right)(2 N-1)|\Lambda|} P_{N}(0),
$$

consequently its mass, as given in eq. (5.7), is also bounded from below.

Next we consider global thermodynamical stability by requiring free energy to be negative. Using eqs. (5.4) and (5.8), we compute free energy,

$$
F=M-T S=\frac{1}{2 d_{0}-2 N+2}\left(P_{N}(0) r_{h}^{2 d_{0}-2 N+1}+(2 N-1) \Lambda r_{h}^{2 d_{0}+1}\right) .
$$

For $d_{0}=N, P_{N}(0)=0$ and $\Lambda<0$ ( $N$ odd), hence free energy is always negative ensuring global stability while for even $N$ and $\Lambda>0$ it is always positive thereby implying instability. That is black hole is thermodynamically unstable for $\Lambda>0$ and $N$ even. Thus 
thermodynamical stability requires $\Lambda<0$ and $N$ odd. When $d_{0}=N$ (pure Lovelock), there is no restriction of any kind while for $d_{0}>N$ for which $P_{N}(0)>0$, there is lower bound on horizon size given by

$$
r_{h}^{2 N}>\frac{P_{N}(0)}{(2 N-1)|\Lambda|},
$$

which already implies, as expected, local thermodynamical stability eq. (5.12).

Note however that our analysis is formal in the following sense. When $d_{0}>N$ the configuration with $M=0$ is not allowed in our formalism as it is forbidden from equations (5.7) and (5.14), since $P_{N}(0)>0$. One could think that in the critical, odd $d_{0}=N$ case, the limit $r_{h} \rightarrow 0$ which sends also $M$ to zero, could be taken without restrictions. And in fact the entropy (5.4) and the temperature (5.8) (with $P_{N}(0)=0$ ) vanish in this limit. But then a naked singularity appears at the origin $r=0$ (see eq. (3.7) in section 3.3) and thus we must keep $M>0$ to prevent this singularity from forming. This means that the global stability analysis must be considered formal.

\section{Discussion}

We have shown, within the framework of Lovelock theories, that the property that the EOM are polynomial, which it is well known to hold in the case of spherically symmetric horizons, can be generalized to horizons that are product of two equal spheres (though now the Weyl tensor is not vanishing), which are a particular case of Einstein manifolds. On the other hand we also know that in the case of Gauss-Bonnet gravity (Lovelock $N=2$ ) this polynomial property of the EOM holds whenever the horizon is an Einstein manifold [14] (thus including the one-sphere, two-spheres cases). It can be conjectured that, in a general Lovelock theory, the polynomial property that we find for two-spheres topology could be extended to the more general case of Einstein horizons. An indication in this direction is the study of $N=3$ Lovelock in [22].

What does two sphere topology entail is that horizon space is non-maximally symmetric Einstein space which has non-zero Weyl curvature, though both Weyl and Riemann have vanishing covariant derivative. Product of spheres cause solid angle deficits which cancel out each-other's contribution to Ricci tensor [21] but not to Riemann tensor. Thus for $N \geq 2$, EOM always has Riemann present and hence contribution to it from solid angle deficit has to be anulled out, and that is done with an additional numerical parameter $p$, as shown in the $N=2$ case, in which it appears under the radical in the solution. It is in fact a measure of non-zero Weyl curvature [14] which is absent for $N=1$. The general feature of vacuum equation, ultimately reducing to an algebraic polynomial is carried through even for two sphere topology for $d_{0} \geq N$. That is why we have been able to solve pure Lovelock vacuum equation for arbitrary order $N$ in this enlarged general setting. Though our general analysis refers to the critical dimension $d_{0}=N$, however the equation reducing to an algebraic polynomial is a general feature true for all $d_{0} \geq N$.

It turns out that in the critical $d_{0}=N$ case, constant $p$ and $\Lambda$ always bear opposite sign, the former is positive or negative according as $N$ is odd or even and opposite is the 
case for $\Lambda$. For even $N, p<0$ and $\Lambda>0$, spacetime has two horizons, black hole and cosmological, and black hole mass is constrained in a range given in terms of $\Lambda$. While for odd $N, \Lambda<0$, there is only one black hole horizon without any restriction. Remarkably thermodynamical stability also has the similar discerning feature. That is spacetime is thermodynamically stable for odd $N$ and $\Lambda<0$ while it is unstable for even $N$ and $\Lambda>0$.

We thus conclude that two sphere topology for pure Lovelock gravity clearly discerns between odd and even Lovelock order $N$, consequently negative and positive $\Lambda$ and it is the former which is favored. This is a remarkable and interesting property of this setting which has been revealed only when we probed higher orders, $N>2$. Let's reiterate that universality of kinematic property of gravity in all odd $d=2 N+1$ dimensions demands the pure Lovelock equation which is valid only for the two $d=2 N+1,2 N+2$ dimensions. This is the main and critical motivation for pure Lovelock gravity. In the context of the present study we therefore always take $d=2 N+2$ which implies $d_{0}=N$.

\section{Acknowledgments}

This work has been supported through grants FPA2013-46570, 2014-SGR-104 and Consolider CPAN. ND wishes to acknowledge Departament ECM Universitat de Barcelona for a kind invitation for a visit that facilitated this work. We also thank the Referee for constructive criticism and useful suggestions.

\section{A Proof that $\left[\mathcal{L}_{N}\right]_{0}$ is a derivative}

Consider equation (2.4) for $\rho=\mu=0$. The factor $\sqrt{-g}$ is, up to irrelevant dependences on the angles, $r^{2 d_{0}}$. Obviously the term with the cosmological constant is an $r$-derivative, so we will only consider in this appendix the expression $\left[\mathcal{L}_{N}\right]_{0}$ with vanishing $\Lambda$,

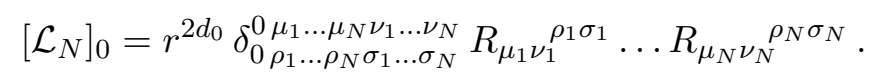

This expresion is a sum of products of $N$ components of the Riemann tensor. We must fill in it the components (2.2). Since the index 0 will not be present in these components, we can only introduce the factors $D(1, i), D\left(1, i^{\prime}\right), D(i, j), D\left(i^{\prime}, j^{\prime}\right), D\left(i, i^{\prime}\right)$. Note that the first two objects in this list contain $A^{\prime}(r)$, whereas the rest only contain $A(r)$. So (A.1) will become the sum of two objects, one of them containing $A^{\prime}(r)$ as a factor and the other without this derivative. To construct each of these objects one must consider all possible distributions of the factors $D$ already mentioned, which is a matter of combinatorics. In the procedure one must take into account the location of each $D$ within any of the $N$ slots available in each additive term and, additionally, the combinatorics associated with the choice of angles, $i, i^{\prime}$, for each $D$. 
All in all, using the quantities (2.11) and (2.10), and defining the new ones,

$$
\begin{aligned}
\bar{C}[m, s] & =\frac{1}{2^{m}} \sum_{k=0}^{m}\left(\begin{array}{c}
m \\
k
\end{array}\right) \frac{s !}{(s-2 m+2 k) !} \frac{(s-1) !}{(s-2 k-1) !}, \\
F_{N}\left(A, A^{\prime}\right) & =N \sum_{l=0}^{N-1}\left(\frac{d_{0} !}{\left(d_{0}-l\right) !}\right)^{2}\left(d_{0}-l\right) \bar{C}\left[N-l-1, d_{0}-l\right]\left(-A^{\prime}\right)(-A)^{l}(1-A)^{N-l-1},
\end{aligned}
$$

An alternative form for $F_{N}\left(A, A^{\prime}\right)$ is:

$$
\begin{aligned}
F_{N}\left(A, A^{\prime}\right)= & \sum_{l=0}^{N-1} \sum_{k=0}^{N-l-1} \frac{1}{2^{N-l-1}} \frac{N !}{k ! l !(N-k-l-1) !} \frac{d_{0} !}{\left(d_{0}-2 k-l-1\right) !} \\
& \frac{d_{0} !}{\left(d_{0}+2 k-2(-l+N-1)-l\right) !}\left(-A^{\prime}(r)\right)(-A(r))^{l}(1-A(r))^{N-l-1}
\end{aligned}
$$

we end up with the result

$$
\frac{1}{4^{N}}\left[\mathcal{L}_{N}\right]_{0}=r^{2 d_{0}-2 N+1} F_{N}\left(A, A^{\prime}\right)+r^{2 d_{0}-2 N} P_{N}(A)
$$

but it turns out that

$$
\frac{d}{d r} P_{N}(A)=\left(2 d_{0}-2 N+1\right) F_{N}\left(A, A^{\prime}\right)
$$

and therefore

$$
\frac{1}{4^{N}}\left[\mathcal{L}_{N}\right]_{0}=\frac{1}{2 d_{0}-2 N+1} \frac{d}{d r}\left(r^{2 d_{0}-2 N+1} P_{N}(A)\right),
$$

which proves our claim.

\section{B Proof of (2.7)}

Here we prove (2.7). To this effect we will consider the Noether identities for gauge theories applied to the Lovelock Lagrangians. These Lagrangians are generally covariant -and hence gauge theories- under the diffeomorphism group (coordinate reparametrizations).

Consider a field theory with dynamics derived from a variational principle with Lagrangian $\mathcal{L}$ and fields $\phi^{A}$ (here $\phi^{A}$ means a generic field or field component). The Noether symmetries (satisfying that $\delta \mathcal{L}$ is a divergence) are implemented by infinitesimal transformations

$$
\delta \phi^{A}=R_{a}^{A} \epsilon^{a}+R_{a}^{A \mu} \partial_{\mu} \epsilon^{a},
$$

with $\epsilon^{a}$ being the infinitesimal parameters of the symmetries, and with the index $a$ running over the number of independent symmetries. The coefficients $R_{a}^{A}, R_{a}^{A \mu}$ are functions of the fields and their derivatives. The gauge character of the symmetries relies in the fact that the functions $\epsilon^{a}$ are arbitrary functions of the coordinates -and even of the fields. If $[\mathcal{L}]_{A}$ are the functional (Euler-Lagrange) derivatives with respect to $\phi^{A}$, the Noether identities are written as

$$
[\mathcal{L}]_{A} R_{a}^{A}-\partial_{\mu}\left([\mathcal{L}]_{A} R_{a}^{A \mu}\right)=0
$$


Applying these identities to the Einstein-Hilbert Lagrangian one obtains the well known doubly contracted Bianchi identities for the Riemann tensor.

For convenience, we will move momentarily to the formalism that uses the vielbein and the Lorentz connection as the independent fields. The vielbein $e_{\mu}^{I}$ extracted from the metric (2.1) is (we use the notation $I=\underline{\nu}$ for the flat indices)

$$
e_{0}^{\underline{0}}=\sqrt{A(r)}, \quad e_{1}^{\frac{1}{1}}=\frac{1}{\sqrt{A(r)}}, \quad e_{i}^{\underline{i}}=r f_{i}, \quad e_{i^{\prime}}^{\frac{i^{\prime}}{}}=r f_{i^{\prime}}
$$

where $f_{i}$ and $f_{i^{\prime}}$ are trigonometric functions of the angles of the spheres whose specific form is irrelevant to us. notice that the vielbein is "diagonal" in its indices.

The Lovelock Lagrangian (with the cosmological constant term) in this formalism will be denoted $\tilde{\mathcal{L}}_{N}$ and its EOM are the functional derivatives with respect to the vielbein and the Lorentz connection. The functional derivatives with respect to the connection are proportional to the torsion tensor and we require this tensor to vanish in order to recover our original setting where the connection was just the Levi Civita connection. Thus we need not worry about the EOM coming from variations of the connection.

There are two types of gauge symmetries in the present formalism, namely diffeomorphism invariance and local Lorentz invariance. The Noether identities for the local Lorentz invariance are empty in our case. So we will focus on diffeomorphism invariance. The vielbein vectors $e_{\mu}^{I}$ transform, under a difeomorphism $\delta x^{\mu}=-\epsilon^{\mu}$, with the Lie derivative

$$
\delta e_{\mu}^{I}=\epsilon^{\rho} \partial_{\rho} e_{\mu}^{I}+e_{\rho}^{I} \partial_{\mu} \epsilon^{\rho}
$$

and thus we infer from (B.1) the identifications

$$
R_{\rho}^{\left(e_{\mu}^{I}\right)}=\partial_{\rho} e_{\mu}^{I}, \quad R_{\rho}^{\left(e_{\mu}^{I}\right) \sigma}=\delta_{\mu}^{\sigma} e_{\rho}^{I} .
$$

For the N-Lovelock Lagrangian the Noether identities (B.2) become

$$
\left[\tilde{\mathcal{L}}_{N}\right]_{\left(e_{\mu}^{I}\right)} R_{\rho}^{\left(e_{\mu}^{I}\right)}-\partial_{\sigma}\left(\left[\tilde{\mathcal{L}}_{N}\right]_{\left(e_{\mu}^{I}\right)} R_{\rho}^{\left(e_{\mu}^{I}\right) \sigma}\right)=0
$$

As an identity, (B.4) holds for any field configuration, and in particular for (B.3) (which implies (2.1)). When we plug (B.3) into (B.4) we can ease the notation because, the vielbein being "diagonal", each contributing field is identified by a $\mu$ index: $e_{\mu}^{\frac{\mu}{\mu}} \rightarrow \mu$, we get

$$
\left[\tilde{\mathcal{L}}_{N}\right]_{\mu} R_{\rho}^{(\mu)}-\partial_{\sigma}\left(\left[\tilde{\mathcal{L}}_{N}\right]_{\mu} R_{\rho}^{(\mu) \sigma}\right)=0 .
$$

It is easy to see that the functional derivatives $\left[\tilde{\mathcal{L}}_{N}\right]_{\mu}$ are proportional to those in (2.4). To be specific, the result is

$$
\left[\tilde{\mathcal{L}}_{N}\right]_{\mu}=\frac{1}{e_{\mu}^{\mu}}\left[\mathcal{L}_{N}\right]_{\mu}
$$

(clearly no sum for $\mu$ ). 
The only nontrivial case for (B.5) is when $\rho=1$ (the radial variable). We obtain, using (2.5), (2.6) and (B.6),

$$
\begin{aligned}
0 & =\left[\tilde{\mathcal{L}}_{N}\right]_{\mu} \partial_{1} e_{\bar{\mu}}^{\underline{\mu}}-\partial_{\mu}\left(\left[\tilde{\mathcal{L}}_{N}\right]_{\mu} e_{1}^{\underline{\mu}}\right)=\left[\tilde{\mathcal{L}}_{N}\right]_{\mu} \partial_{1} e^{\underline{\mu}}-\partial_{1}\left(\left[\tilde{\mathcal{L}}_{N}\right]_{1} e_{1}^{\underline{1}}\right) \\
& =\left[\tilde{\mathcal{L}}_{N}\right]_{i} \partial_{1} e_{i}^{\underline{i}}+\left[\tilde{\mathcal{L}}_{N}\right]_{i^{\prime}} \partial_{1} e_{i^{\prime}}^{i^{\prime}}+\left[\tilde{\mathcal{L}}_{N}\right]_{0} \partial_{1} e_{0}^{\underline{0}}+\left[\tilde{\mathcal{L}}_{N}\right]_{1} \partial_{1} e_{1}^{\underline{1}}-\partial_{1}\left(\left[\tilde{\mathcal{L}}_{N}\right]_{1} e_{1}^{\underline{1}}\right) \\
& =\left[\tilde{\mathcal{L}}_{N}\right]_{i} f_{i}+\left[\tilde{\mathcal{L}}_{N}\right]_{i^{\prime}} f_{i^{\prime}}+\left[\tilde{\mathcal{L}}_{N}\right]_{0} \partial_{1} e_{0}^{\underline{0}}-\partial_{1}\left(\left[\tilde{\mathcal{L}}_{N}\right]_{1}\right) e_{1}^{\underline{1}} \\
& =\frac{2 d_{0}}{r}\left[\mathcal{L}_{N}\right]_{i}+\frac{1}{\sqrt{A(r)}}\left[\mathcal{L}_{N}\right]_{0} \partial_{1} \sqrt{A(r)}-\partial_{1}\left(\left[\mathcal{L}_{N}\right]_{1} \sqrt{A(r)}\right) \frac{1}{\sqrt{A(r)}} \\
& =\frac{2 d_{0}}{r}\left[\mathcal{L}_{N}\right]_{i}-\partial_{1}\left(\left[\mathcal{L}_{N}\right]_{0}\right) .
\end{aligned}
$$

which is the result (2.7).

Open Access. This article is distributed under the terms of the Creative Commons Attribution License (CC-BY 4.0), which permits any use, distribution and reproduction in any medium, provided the original author(s) and source are credited.

\section{References}

[1] N. Dadhich, S.G. Ghosh and S. Jhingan, The Lovelock gravity in the critical spacetime dimension, Phys. Lett. B 711 (2012) 196 [arXiv:1202.4575] [INSPIRE].

[2] N. Dadhich, Characterization of the Lovelock gravity by Bianchi derivative, Pramana $\mathbf{7 4}$ (2010) 875 [arXiv: 0802.3034] [INSPIRE].

[3] D.G. Boulware and S. Deser, String generated gravity models, Phys. Rev. Lett. 55 (1985) 2656 [INSPIRE].

[4] J.T. Wheeler, Symmetric solutions to the maximally Gauss-Bonnet extended Einstein equations, Nucl. Phys. B 273 (1986) 732 [INSPIRE].

[5] J.T. Wheeler, Symmetric solutions to the Gauss-Bonnet extended Einstein equations, Nucl. Phys. B 268 (1986) 737 [INSPIRE].

[6] B. Whitt, Spherically symmetric solutions of general second order gravity, Phys. Rev. D 38 (1988) 3000 [INSPIRE].

[7] M. Bañados, C. Teitelboim and J. Zanelli, Dimensionally continued black holes, Phys. Rev. D 49 (1994) 975 [gr-qc/9307033] [INSPIRE].

[8] J. Crisostomo, R. Troncoso and J. Zanelli, Black hole scan, Phys. Rev. D 62 (2000) 084013 [hep-th/0003271] [INSPIRE].

[9] N. Dadhich, J.M. Pons and K. Prabhu, Thermodynamical universality of the Lovelock black holes, Gen. Rel. Grav. 44 (2012) 2595 [arXiv:1110.0673] [InSPIRE].

[10] X.O. Camanho and J.D. Edelstein, A Lovelock black hole bestiary, Class. Quant. Grav. 30 (2013) 035009 [arXiv:1103.3669] [INSPIRE].

[11] H. Maeda, S. Willison and S. Ray, Lovelock black holes with maximally symmetric horizons, Class. Quant. Grav. 28 (2011) 165005 [arXiv:1103.4184] [inSPIRE]. 
[12] H. Nariai, On some static solutions of Einstein's gravitational field equations in a spherically symmetric case, Sci. Rep. Tohoku Univ. 34 (1950) 160.

[13] H. Nariai, On a new cosmological solution of Einstein's field equations of gravitation, Sci. Rep. Tohoku Univ. 35 (1951) 62.

[14] G. Dotti and R.J. Gleiser, Obstructions on the horizon geometry from string theory corrections to Einstein gravity, Phys. Lett. B 627 (2005) 174 [hep-th/0508118] [INSPIRE].

[15] C. Bogdanos, C. Charmousis, B. Gouteraux and R. Zegers, Einstein-Gauss-Bonnet metrics: black holes, black strings and a staticity theorem, JHEP 10 (2009) 037 [arXiv:0906.4953] [INSPIRE].

[16] G. Dotti, J. Oliva and R. Troncoso, Vacuum solutions with nontrivial boundaries for the Einstein-Gauss-Bonnet theory, Int. J. Mod. Phys. A 24 (2009) 1690 [arXiv:0809.4378] [INSPIRE].

[17] G. Dotti, J. Oliva and R. Troncoso, Static solutions with nontrivial boundaries for the Einstein-Gauss-Bonnet theory in vacuum, Phys. Rev. D 82 (2010) 024002 [arXiv: 1004.5287] [INSPIRE].

[18] H. Maeda, Gauss-Bonnet black holes with non-constant curvature horizons, Phys. Rev. D 81 (2010) 124007 [arXiv: 1004.0917] [INSPIRE].

[19] J.M. Pons and N. Dadhich, On static black holes solutions in Einstein and Einstein-Gauss-Bonnet gravity with topology $\mathrm{SO}(N) \times \mathrm{SO}(N)$, arXiv:1408.6754 [INSPIRE].

[20] N. Dadhich, J.M. Pons and K. Prabhu, On the static Lovelock black holes, Gen. Rel. Grav. 45 (2013) 1131 [arXiv:1201.4994] [INSPIRE].

[21] B. Kol, Topology change in general relativity and the black hole black string transition, JHEP 10 (2005) 049 [hep-th/0206220] [INSPIRE].

[22] N. Farhangkhah and M. Dehghani, Lovelock black holes with nonmaximally symmetric horizons, Phys. Rev. D 90 (2014) 044014 [arXiv:1409.1410] [INSPIRE]. 